\title{
Comment on "Intervention Effects of a School-Based Health Promotion Programme on Obesity Related Behavioural Outcomes"
}

\author{
Peng Li, ${ }^{1}$ Andrew W. Brown, ${ }^{1}$ J. Michael Oakes, ${ }^{2}$ and David B. Allison ${ }^{1}$ \\ ${ }^{1}$ Office of Energetics and Nutrition Obesity Research Center, University of Alabama at Birmingham, Birmingham, AL 35294, USA \\ ${ }^{2}$ Division of Epidemiology and Minnesota Population Center, University of Minnesota, Minneapolis, MN 55454, USA \\ Correspondence should be addressed to David B. Allison; dallison@uab.edu
}

Received 17 December 2014; Accepted 18 May 2015

Academic Editor: Bernhard H. Breier

Copyright (C) 2015 Peng Li et al. This is an open access article distributed under the Creative Commons Attribution License, which permits unrestricted use, distribution, and reproduction in any medium, provided the original work is properly cited.

The paper, "Intervention Effects of a School-Based Health Promotion Programme on Obesity Related Behavioural Outcomes" by Kobel et al. [1], reports secondary outcomes from a cluster-randomized controlled trial (cRCT): the Baden-Württemberg primary school study (DRKS-ID: DRKS00000494) [2]. Importantly, the design of this cRCT properly incorporated crucial aspects of such trials, such as the lack of independence of subjects within clusters and the nesting of clusters within treatment conditions [2]. Additionally, plausible analytical models (e.g., linear mixed effects models or GEE models) were planned [2]. Unfortunately, the statistical analysis ultimately reported in [1] is inconsistent with the predefined analysis plan and does not take the impact of clustering and nesting into account. Ignoring the potential similarity among individuals in the same cluster (school) can underestimate the variance of intervention effects and inflate the degrees of freedom in the hypothesis testing and, therefore, increase the type I error rates and jeopardize the validity of conclusions from cRCTs $[3,4]$.

We agree with others that the cRCT is one of the most important designs in the community- or school-based obesity studies. Unfortunately, many seem not to understand the implications of clustering and nesting and the need for specialized statistical models to analyze such data. To be consistent with recommended best practices and to improve the transparency and utility of cRCTs, we suggest that Kobel et al. reanalyze their data according to their prespecified plan by taking the clustering and nesting into account and report their results following the CONSORT 2010: extension to cluster-randomized trials [5].

\section{Disclaimer}

The opinions expressed are those of the authors and do not necessarily represent those of the NIH or any other organization.

\section{Conflict of Interests}

The University of Alabama at Birmingham has received unrestricted gifts, grants, and contracts from companies which market soft drinks and breakfast foods, including PepsiCo and The Coca-Cola Company. David B. Allison has received consulting fees from the Kellogg Company.

\section{Acknowledgment}

This paper is supported in part by NIH Grants R25HL124208 and P30DK056336.

\section{References}

[1] S. Kobel, T. Wirt, A. Schreiber et al., "Intervention effects of a school-based health promotion programme on obesity related 
behavioural outcomes," Journal of Obesity, vol. 2014, Article ID 476230, 8 pages, 2014.

[2] J. Dreyhaupt, B. Koch, T. Wirt et al., "Evaluation of a health promotion program in children: study protocol and design of the cluster-randomized Baden-Württemberg primary school study [DRKS-ID: DRKS00000494]," BMC Public Health, vol. 12, no. 1, article 157, 2012.

[3] D. M. Murray, S. P. Varnell, and J. L. Blitstein, "Design and analysis of group-randomized trials: a review of recent methodological developments," American Journal of Public Health, vol. 94, no. 3, pp. 423-432, 2004.

[4] M. J. Campbell, A. Donner, and N. Klar, "Developments in cluster randomized trials and Statistics in Medicine," Statistics in Medicine, vol. 26, no. 1, pp. 2-19, 2007.

[5] M. K. Campbell, G. Piaggio, D. R. Elbourne, and D. G. Altman, "Consort 2010 statement: extension to cluster randomised trials," British Medical Journal, vol. 345, article e5661, 2012. 


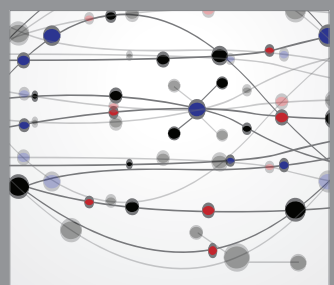

The Scientific World Journal
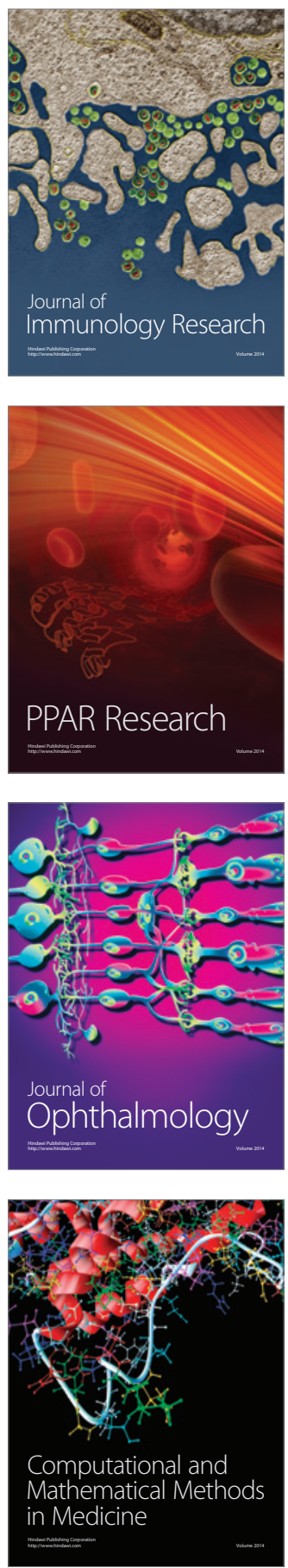

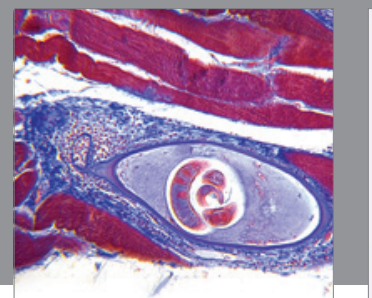

Gastroenterology

Research and Practice
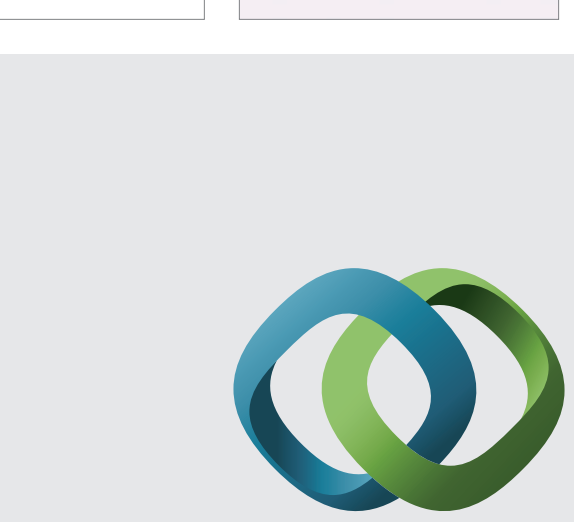

\section{Hindawi}

Submit your manuscripts at

http://www.hindawi.com

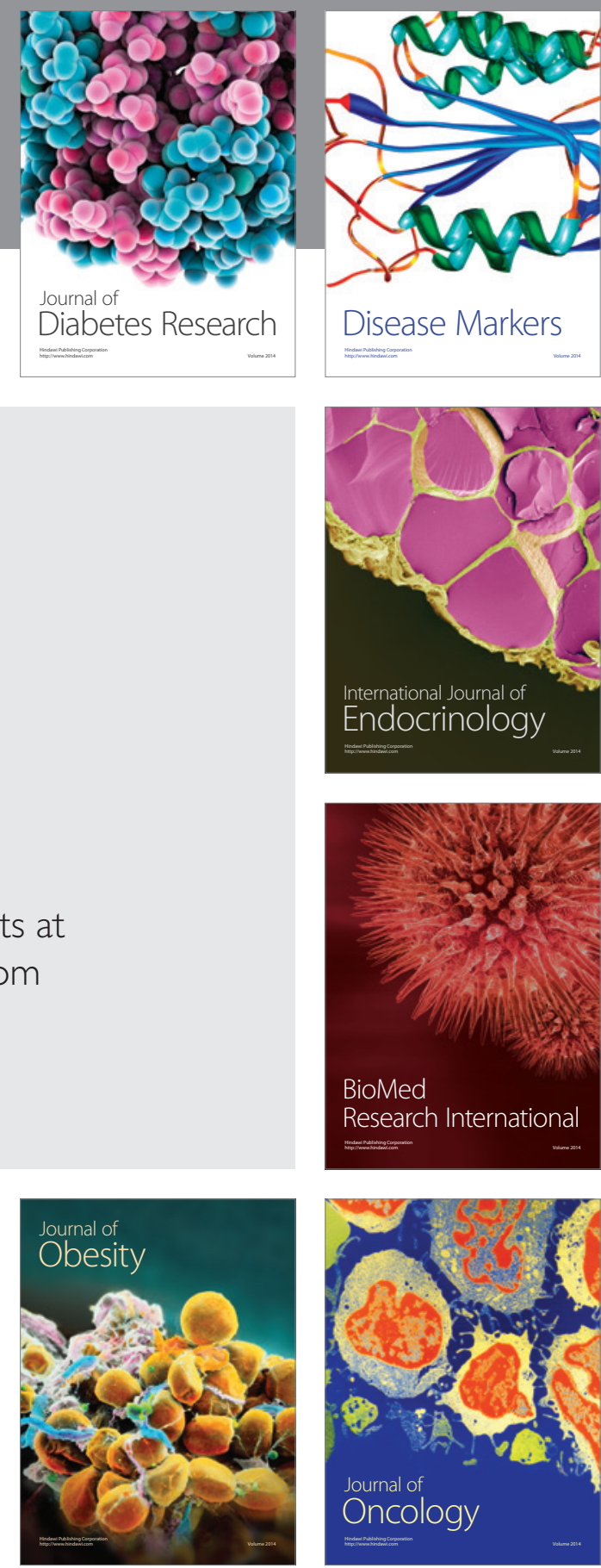

Disease Markers
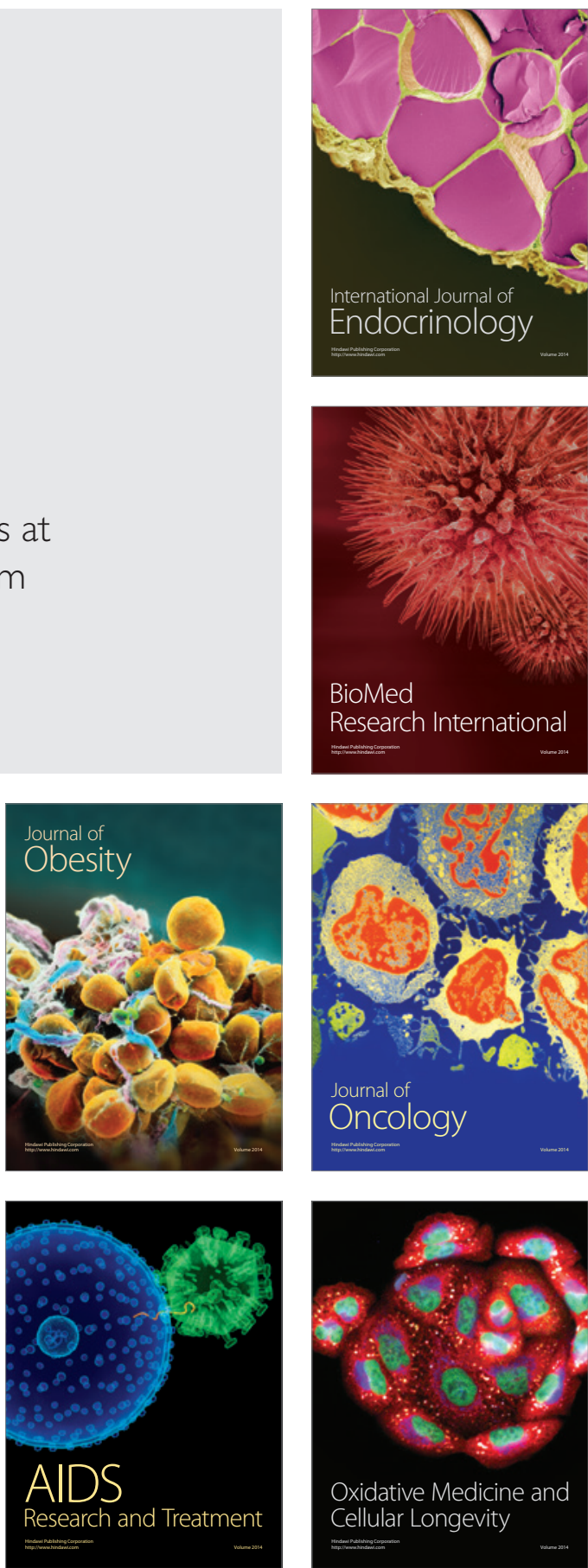